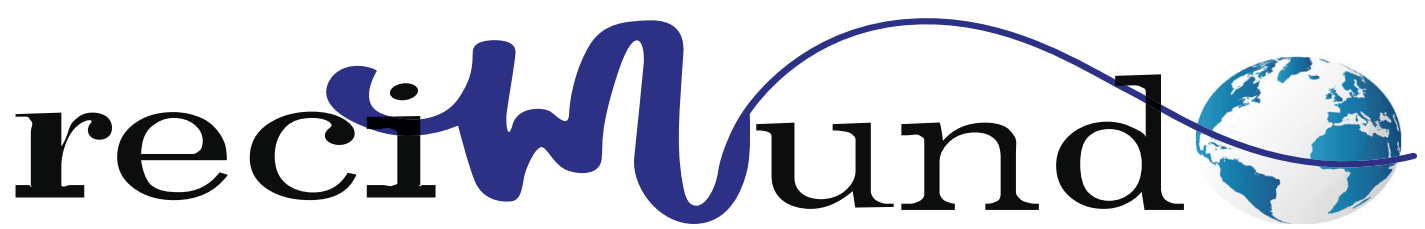

Revista Científica Mundo de la Investigación y el Conocimiento

DOI: 10.26820/recimundo/4.(4).octubre.2020.539-547

URL: http://recimundo.com/index.php/es/article/view/1011

EDITORIAL: Saberes del Conocimiento

REVISTA: RECIMUNDO

ISSN: 2588-073X

TIPO DE INVESTIGACióN: Artículo de Revisión

CÓDIGO UNESCO: 32 Ciencias Médicas

PAGINAS: $539-547$

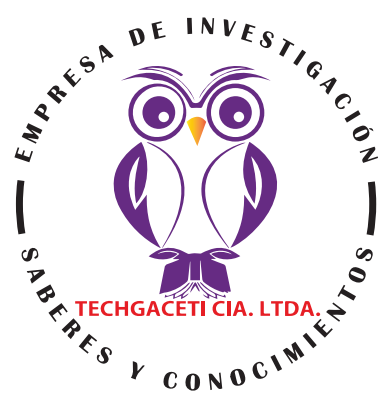

\title{
Causas y consecuencias de los pacientes diagnosticados con hepatitis crónica
}

Causes and consequences of patients diagnosed with chronic hepatitis

Causas e consequências dos pacientes diagnosticados com hepatite crónica

Andrea Natacha Pico Tagle'; Luis Gustavo Chávez Heredia; Luis Antonio Moreira Moreira3;

Juliana Edith Palacios Saltos ${ }^{4}$

RECIBIDO: 10/11/2020 ACEPTADO: 26/11/2020 PUBLICADO: 13/12/2020

1. Médica Cirujana; Médico General en Funciones Hospitalarias; Hospital Instituto Ecuatoriano de Seguridad Social; Manta, Ecuador; natachapicotagle@hotmail.com; https://orcid.org/0000-0003-4370-3630

2. Médico Cirujano; Médico General en Funciones Hospitalarias; Hospital Instituto Ecuatoriano de Seguridad Social; Manta, Ecuador; drgusy@gmail.com; https://orcid.org/0000-0002-4257-9845

3. Médico Cirujano; Médico General en Funciones Hospitalarias; Hospital General Rodriguez Zambrano; Manta, Ecuador; luis.a.moreiramedico21@gmail.com; https://orcid.org/0000-0003-3353-1126

4. Médica Cirujana; Médico General en Funciones Hospitalarias; Hospital Instituto Ecuatoriano de Seguridad Social; Manta, Ecuador; julianapalacios_27@hotmail.com; https://orcid.org/0000-0001-6288-2625

CORRESPONDENCIA

Andrea Natacha Pico Tagle

natachapicotagle@hotmail.com

Manta, Ecuador

๑ RECIMUNDO; Editorial Saberes del Conocimiento, 2020 


\section{RESUMEN}

La hepatitis crónica se define como una enfermedad hepática crónica difusa que existe durante al menos 6 meses dejando como secuela la cirrosis. Es de etiología múltiple, por lo que es fundamental la realización de una biopsia hepática para el diagnóstico y pronóstico. La hepatitis crónica relacionada con la hepatitis $\mathrm{B}$ es de progresión lenta. No se recomienda la terapia con corticoides, en este caso, la terapia antiviral actual convierte al paciente positivo al antígeno e de la hepatitis B en anti-HBe en aproximadamente un $50 \%$. La hepatitis crónica relacionada con la hepatitis por virus no A y no B carecen de la falta de un marcador de diagnóstico, sin embargo, se tiene que ninguna terapia actual tiene beneficios comprobados. Esta enfermedad presenta un cuadro bioquímico e inmunológico muy activo, es también relacionada con fármacos por lo cual se recomienda la retirada de la droga para la recuperación del paciente, ya que, las muertes generalmente se producen debido a la continuación de la misma. Se detallan las indicaciones de progresión a un estado terminal con probabilidad de supervivencia de menos de 6 meses. Estos son útiles para decidir sobre el trasplante hepático antes de que el paciente esté moribundo.

Palabras clave: Hepatitis, Hepatitis Crónica, Cirrosis, Trasplante Hepático.

\section{ABSTRACT}

Chronic hepatitis is defined as a diffuse chronic liver disease that exists for at least 6 months, leaving cirrhosis as a sequel. It is of multiple etiology, so a liver biopsy is essential for diagnosis and prognosis. Chronic hepatitis B related to hepatitis B is slow to progress. Corticosteroid therapy is not recommended; in this case, current antiviral therapy converts the patient positive for hepatitis $B$ e antigen into anti-HBe by approximately $50 \%$. Chronic hepatitis related to non-A and non-B viral hepatitis lack a lack of a diagnostic marker, however, no current therapy has proven benefits. This disease presents a very active biochemical and immunological picture, it is also related to drugs, for which the withdrawal of the drug is recommended for the recovery of the patient, since deaths generally occur due to its continuation. Indications of progression to a terminal state with a probability of survival of less than 6 months are detailed. These are helpful in deciding about liver transplantation before the patient is dying.

Keywords: Hepatitis, Chronic Hepatitis, Cirrhosis, Liver Transplantation.

\section{RESUMO}

A hepatite crónica é definida como uma doença hepática crónica difusa que existe há pelo menos 6 meses, deixando a cirrose como uma sequela. É de etiologia múltipla, pelo que uma biopsia hepática é essencial para o diagnóstico e prognóstico. A hepatite B crónica relacionada com a hepatite $\mathrm{B}$ é lenta a progredir. A terapia com corticosteroides não é recomendada; neste caso, a actual terapia antiviral converte o paciente positivo para a hepatite B e antigénio em anti-HBe em aproximadamente $50 \%$. A hepatite crónica relacionada com hepatite não-A e não-B viral carece de um marcador de diagnóstico, contudo, nenhuma terapia actual tem benefícios comprovados. Esta doença apresenta um quadro bioquímico e imunológico muito activo, está também relacionada com medicamentos, para os quais a retirada do medicamento é recomendada para a recuperação do paciente, uma vez que as mortes ocorrem geralmente devido à sua continuação. Indicações de progressão para um estado terminal com uma probabilidade de sobrevivência inferior a 6 meses são detalhadas. Estas são úteis para decidir sobre o transplante do fígado antes de o paciente morrer.

Palavras-chave: Hepatite, Hepatite Crónica, Cirrose, Transplante De Fígado. 


\section{Introducción}

La hepatitis crónica es un término utilizado para describir una amplia variedad de afecciones caracterizadas por inflamación crónica del parénquima hepático o lesión de los hepatocitos que da como resultado índices elevados de función hepática. En general, la hepatitis se clasifica como crónica o crónica según la duración de la inflamación y la agresión al parénquima hepático.

Si el período de inflamación o lesión hepatocelular dura menos de seis meses, caracterizado por la normalización de las pruebas de función hepática, se denomina hepatitis aguda. Por el contrario, si la inflamación o la lesión hepatocelular persiste más allá de los seis meses, se denomina hepatitis crónica. (Agrawal, Dhiman, \& Limdi, 2016)

La causa infecciosa más común de hepatitis se debe a una infección viral (hepatitis viral aguda). Sin embargo, la hepatitis crónica también puede ser el resultado de una amplia variedad de causas no infecciosas que incluyen, entre otras, "las drogas (hepatitis inducida por drogas), el alcohol (hepatitis alcohólica), inmunológicas (hepatitis autoinmune, colangitis biliar primaria) o como resultado de daño indirecto secundario a disfunción del tracto biliar (hepatitis colestásica), disfunción hepática relacionada con el embarazo, shock o enfermedad metastásica" (Hosseini, Shor, \& Szabo, 2019). La hepatitis crónica se define como una reacción inflamatoria crónica en el hígado, según lo muestran las pruebas de función hepática y la histología y que continúa sin mejorar durante al menos seis meses. "Morfológicamente, las dos variedades de hepatitis crónica persistente y crónica agresiva (esta última mejor llamada como hepatitis crónica activa) puede describirse como 'enfermedad hepática activa crónica" (Agrawal, Dhiman, \& Limdi, 2016).

Su clasificación se basa en gran medida en evidencia morfológica, que no siempre está disponible; además, esta evidencia suele provenir de pequeñas biopsias con aguja. Estos sufren mucho de errores de muestreo, particularmente en pacientes con hepatitis crónica y cirrosis. Es posible mostrar las apariciones de una hepatitis crónica persistente en una parte y en otra crónica activa. "La interpretación de los cambios morfológicos, particularmente en relación con la fibrosis y la regeneración nodular, exige un patólogo experto, familiarizado con las apariencias de las biopsias hepáticas con aguja" (Agrawal, Dhiman, \& Limdi, 2016).

La distinción entre hepatitis crónica persistente y crónica activa también es difícil debido a la falta de uniformidad de la afectación hepática. Se puede adoptar una opinión falsamente optimista si la biopsia se realiza mientras el paciente está recibiendo grandes dosis de prednisolona. Por tal motivo, a través del siguiente desarrollo investigativo, se presenta una revisión bibliográfica que expone las causas y consecuencias de aquellos pacientes que presentan este tipo de afección, en aras de brindar información de interés para futuras investigaciones relacionadas con temas similares al descrito.

\section{Metodología}

Para el desarrollo de este proceso investigativo, se plantea como metodología la encaminada hacia una orientación científica particular que se encuentra determinada por la necesidad de indagar en forma precisa y coherente una situación, en tal sentido Davila, (2015) define la metodología "como aquellos pasos anteriores que son seleccionados por el investigador para lograr resultados favorables que le ayuden a plantear nuevas ideas" (p.66)

Lo citado por el autor, lleva a entender que el desarrollo de la acción investigativa busca simplemente coordinar acciones enmarcadas en una revisión bibliográfica con el fin de complementar ideas previas relacionadas Causas y consecuencias de los pa- 
cientes diagnosticados con hepatitis crónica a través de una revisión de literatura, para así finalmente elaborar un cuerpo de consideraciones generales que ayuden a ampliar el interés propuesto.

\section{Tipo de Investigación}

Dentro de toda práctica investigativa, se precisan acciones de carácter metodológico mediante las cuales se logra conocer y proyectar los eventos posibles que la determinan. En este sentido, la presente investigación corresponde al tipo documental, definido por Castro (2016), "se ocupa del estudio de problemas planteados a nivel teórico, la información requerida para abordarlos se encuentra básicamente en materiales impresos, audiovisuales y / o electrónicos". (p.41).

En consideración a esta definición, la orientación metodológica incluye la oportunidad de cumplir con una serie de actividades inherentes a la revisión y lectura de diversos documentos, donde se encuentran ideas explicitas relacionadas con los tópicos encargados de identificar una característica inmersa en el estudio. Por lo tanto, se realizaron continuas interpretaciones con el claro propósito de revisar aquellas apreciaciones propuestas por diferentes investigadores en relación al tema de interés, para luego dar la respectiva argumentación a los planteamientos, en función a las necesidades encontradas en la investigación, apoyados en las herramientas tecnológicas para la búsqueda de trabajos con valor científico disponibles en la web que tenían conexión con el objetivo principal de la investigación.

\section{Fuentes Documentales}

El análisis correspondiente a las características que predomina en el tema seleccionado, llevan a incluir diferentes fuentes documentales encargadas de darle el respectivo valor científico y en ese sentido cumplir con la valoración de los hechos a fin de gene- rar nuevos criterios que sirven de referencia a otros procesos investigativos. Para Castro,(2016) las fuentes documentales incorporadas en la investigación documental o bibliográfica, "representa la suma de materiales sistemáticos que son revisados en forma rigurosa y profunda para llegar a un análisis del fenómeno" (p.41). Por lo tanto, se procedió a cumplir con la lectura previa determinada para encontrar aquellos aspectos estrechamente vinculados con el tema, con el fin de explicar mediante un desarrollo las respectivas apreciaciones generales de importancia.

\section{Técnicas para la Recolección de la Infor- mación}

La conducción de la investigación para ser realizada en función a las particularidades que determinan a los estudios documentales, tiene como fin el desarrollo de un conjunto de acciones encargadas de llevar a la selección de técnicas estrechamente vinculadas con las características del estudio. Bolívar, (2015), refiere, que es "una técnica particular para aportar ayuda a los procedimientos de selección de las ideas primarias y secundarias". (p.71).

Tal como lo expresa, Bolívar, (2015) "Las técnicas documentales proporcionan las herramientas esenciales y determinantes para responder a los objetivos formulados y llegar a resultados efectivos" (p. 58). Es decir, para responder con eficiencia a las necesidades investigativas, se introdujeron como técnica de recolección el método inductivo, que hizo posible llevar a cabo una valoración de los hechos de forma particular para llegar a la explicación desde una visión general. El autor Bolívar, (2015) tambien expresa que las técnicas de procesamiento de datos en los estudios documentales "son las encargadas de ofrecer al investigador la visión o pasos que deben cumplir durante su ejercicio, cada una de ellas debe estar en correspondencia con el nivel a emplear" (p. 123). Esto indica, que para llevar a cabo 
el procesamiento de los datos obtenidos una vez aplicadas las técnicas seleccionadas, tales como: fichas de resumen, textual, registros descriptivos entre otros, los mismos se deben ajustar al nivel que ha sido seleccionado.

\section{Resultados}

\section{Etiología}

La inflamación hepática crónica se genera por muchas causas infecciosas y no infecciosas, de las cuales las causas más comunes son secundarias a una infección viral o una lesión hepática inducida por fármacos. Los autores Sosa \& Arrese, (2017) expresan una lista de causas comunes de hepatitis crónica e insuficiencia hepática aguda.

Causas infecciosas:

Virus hepatotrópicos:

o Virus de la hepatitis A (VHA)

o Virus de la hepatitis $B(V H B)$

$\mathrm{O} \quad$ Virus de la hepatitis $\mathrm{C}(\mathrm{VHC})$

o Virus de la hepatitis D (HDV)

o Virus de la hepatitis E (HEV)

Virus no hepatotrópico:

o Virus de Epstein-Barr (EBV)

o Citomegalovirus (CMV)

o Virus del herpes simple (HSV)

o Virus Coxsackie

o Adenovirus

o Virus del dengue

o Coronavirus-19 (COVID-19)

Bacterias, hongos y parásitos.

Las causas relacionadas con toxinas o sustancias incluyen:

- Relacionado con el alcohol: enfermedad del hígado graso, hepatitis alcohólica cronicao cirrosis alcohólica

- Drogas y toxinas o Depende de la dosis, por ejemplo, acetaminofén (paracetamol)

o Reacción farmacológica no dependiente de la dosis, p. Ej., Idiosincrásica relacionada con mayor frecuencia con antibióticos y anticonvulsivos, pero también con estatinas, AINE, suplementos herbales / nutricionales

o Otras toxinas, p. Ej., Hongos (Amanita phalloides), suplementos dietéticos y de hierbas, tetracloruro de carbono, picadura de anémona de mar.

Condiciones inmunológicas o inflamatorias

- Hepatitis autoinmune

- Enfermedad biliar como colangitis biliar primaria o colangitis esclerosante primaria.

Metabólico o hereditario

- Enfermedad del hígado graso no alcohólico

- Hemocromatosis

- Enfermedad de Wilson

Relacionado con el embarazo

- Preeclampsia

- Hígado graso agudo del embarazo

- Síndrome de HELLP

Isquémico y Vascular

- Choque cardiogénico / distributivo

- Hipotension

- Golpe de calor

- Cocaína, metanfetamina, efedrina

- Síndrome de Budd-Chiari agudo

- Síndrome de obstrucción sinusoidal

Diverso

- Hígado graso agudo del embarazo

- Malignidad

- Eclampsia

- Síndrome de HELLP

- Síndrome de reye 
- No funciona el injerto primario después de un trasplante de hígado

\section{Histopatología}

La histopatología de la hepatitis crónica está determinada por la etiología subyacente que causa la lesión hepatocelular. La hepatitis crónica secundaria a sobredosis de acetaminofén muestra características histológicas como necrosis puente central a central e infiltrados de células mínimas inflamatorias.

Las características histopatológicas de la hepatitis cronicasecundaria a infecciones virales suelen mostrar inclusiones virales intranucleares y neutrófilos circundantes. Las características históricas clásicas de la hepatitis autoinmune demuestran inflamación portal y hepatitis de interfaz conocida formalmente como necrosis por etapas, que es esencialmente la presencia de células inflamatorias portal entre el parénquima portal y el hígado (Kwong \& Meyerson, 2019).

Los autores Caballeríaa, Caballeríaa, \& Parésa, (2000) afirman que la esteatosis microvesicular difusa, los cuerpos de Mallory, fibrosis o cirrosis son los hallazgos típicos observados en la lesión hepática relacionada con el alcohol. "La acumulación de hierro con pigmento de hemosiderina hepatocelular y el aumento de las concentraciones de cobre hepático y las muestras de biopsia hepática son los hallazgos histopatológicos clásicos en pacientes con hemocromatosis hereditaria y enfermedad de Wilson, respectivamente" (Semin Diagn Pathol., 2019)

\section{Presentación clínica}

La presentación clínica de la hepatitis crónica depende de la etiología subyacente. Puede manifestarse clínicamente con varios signos y síntomas clínicos, que van desde pruebas de función hepática elevadas asintomáticas hasta insuficiencia hepática crónica que requiere trasplante de hígado.
Por lo tanto, determinar la etiología de la hepatitis crónica es de suma importancia en su manejo clínico, para obtener una historia detallada que incluya la duración de la enfermedad presente, el historial de viajes y la evaluación de actividades de alto riesgo como el uso de drogas intravenosas, consumo de alcohol, antecedentes sexuales, antecedentes de transfusión de hemoderivados o ingesta reciente de alimentos. (Sosa \& Arrese, 2017)

También es necesario que el historial de medicamentos incluya no solo medicamentos recetados recientes o actuales, sino también medicamentos de venta libre, acetaminofén (paracetamol). Los pacientes con hepatitis viral crónica suelen presentar "síntomas como fiebre, malestar general, fatiga, pérdida de apetito, vómitos, diarrea y dolor abdominal. Los pacientes también pueden informar decoloración amarillenta de la esclerótica (ictericia) y / o piel (ictericia), orina de color oscuro y heces de color claro" (Hosseini, Shor, \& Szabo, 2019)

Dependiendo de la etiología subyacente, los hallazgos del examen físico pueden variar "desde la presencia de ictericia e ictericia hasta signos de encefalopatía aguda, convulsiones, diátesis hemorrágica, hipotensión y otras manifestaciones relacionadas con insuficiencia multiorgánica" (Montrief \& Koyfman, 2019). En pacientes que presentan enfermedad hepática cronicao crónica pueden observarse signos de enfermedad hepática crónica, como caput medusae, nevos araña, eritema palmar, ascitis, contractura de Dupuytren, ginecomastia y encefalopatía hepática.

\section{Evaluación}

Al evaluar a los pacientes con hepatitis aguda, es muy importante distinguir entre hepatitis crónica y hepatitis crónica. Las pruebas bioquímicas como "AST, ALT, fosfatasa alcalina, GGT, lactato deshidrogenasa, bilirrubina, PT / INR y albúmina determinan el fun- 
cionamiento normal del hígado y cualquier anomalía en estas pruebas es indicativa de lesión de los hepatocitos por causas infecciosas y no infecciosas" (Agrawal, Dhiman, \& Limdi, 2016)

De acuerdo a lo descrito anteriormente, la posible etiología y gravedad de la lesión hepatocelular "se puede determinar en función de la anomalía de una o más de estas pruebas bioquímicas que están involucradas en el desempeño de una función hepática específica" (Woreta, 2014). Además, es muy importante mantener la sospecha de un proceso extrahepático que podría estar contribuyendo a pruebas de función hepática anormales como embarazo, acidosis láctica, sepsis y disfunción cardíaca.

\section{Tratamiento / Manejo}

El tratamiento de la hepatitis crónica depende del factor etiológico específico implicado en la lesión crónica de los hepatocitos. Las hepatitis A y E son las causas infecciosas más frecuentes de hepatitis crónica y suelen tener un curso clínico autolimitado, resolviéndose en 2 a 4 semanas con tratamiento de apoyo que incluye líquidos intravenosos, antieméticos y tratamiento sintomático." Los pacientes deben evitar el uso de alcohol y otros medicamentos potencialmente hepatotóxicos y suplementos de venta libre, pero de lo contrario. También deben recibir educación sobre cómo reducir el riesgo de transmisión de la infección a otras personas" (Caballeríaa, Caballeríaa, \& Parésa, 2000).

La ingesta reglar de acetaminofén es una causa común no infecciosa de hepatitis crónica que conduce a insuficiencia hepática crónica y debe tenerse en cuenta en todos los pacientes que presentan signos y síntomas de insuficiencia. "El tratamiento oportuno con $\mathrm{N}$-acetilcisteína debe iniciarse lo antes posible después de obtener una historia inicial y una prueba de acetaminofén. La N-acetilcisteína se puede administrar por vía oral o intravenosa según el escenario clínico que se menciona a continuación" De acuerdo con Montrief \& Koyfman, (2019) los protocolo para el uso de N- acetilcisteína es el siguiente:

- Protocolo oral de 72 horas- N-acetilcisteína oral: 140 mg / kg por vía oral como dosis de carga, seguido de $70 \mathrm{mg} / \mathrm{kg}$ cada 4 horas para un total de 17 dosis

- Protocolo intravenoso de 20 horas $\mathrm{N}$-acetilcisteína IV: $150 \mathrm{mg} / \mathrm{kg}$ por vía intravenosa durante 60 minutos como dosis de carga, seguido de $50 \mathrm{mg} / \mathrm{kg}$ durante 4 horas (12,5 mg / k/ por hora durante 4 horas), luego $100 \mathrm{mg} / \mathrm{kg}$ durante 16 horas ( 6,25 $\mathrm{mg} / \mathrm{kg}$ por hora durante 16 horas)

El tratamiento con $\mathrm{N}$-acetilcisteína también "se recomienda para todos los pacientes con insuficiencia hepática aguda, excepto hepatitis isquémica, con o sin evidencia de sobredosis de acetaminofén" (Montrief \& Koyfman, 2019). La mayoría de los pacientes con síntomas mínimos y pruebas de función hepática anormales y función hepática sintética normal pueden ser evaluados de forma ambulatoria o remitidos a hepatología.

En pacientes con "función hepática elevada persistentemente sin una identificación clara de una etiología específica, se justifica una evaluación adicional con una biopsia hepática" (Woreta, 2014). Con poca frecuencia, los pacientes con hepatitis crónica asociada con insuficiencia hepática crónica caracterizada por encefalopatía y coagulopatía hepática (INR mayor de 1,5) deben ser analizados y evaluados por el equipo de hepatología para su posible traslado al centro de trasplante de hígado más cercano.

\section{Diagnóstico diferencial}

Además de evaluar las causas antes mencionadas de hepatitis crónica causada por lesión directa de los hepatocitos, también

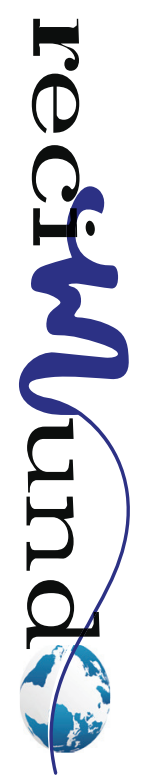


"se deben considerar otras afecciones que causan lesión secundaria de etiologías extrahepáticas o no hepáticas en el diagnóstico diferencial que incluye coledocolitiasis, neoplasias biliares o pancreáticas, metástasis hepáticas, sepsis, sistémica. hipotensión, trombosis de la arteria hepática, insuficiencia cardíaca congestiva, etc" (Montrief \& Koyfman, 2019).

\section{Pronóstico}

El pronóstico de la hepatitis aguda o crónica depende de la etiología que causa la lesión directa de los hepatocitos. La identificación oportuna del agente etiológico que causa la hepatitis crónica y el manejo específico es de suma importancia para reducir la morbilidad y la mortalidad.

\section{Complicaciones}

Aunque es poco común, la complicación más grave de la hepatitis crónica es "la progresión a insuficiencia hepática crónica (IHA) que se caracteriza por un aumento de 2-3 veces en las transaminasas séricas, hiperbilirrubinemia, coagulopatía y encefalopatía hepática de inicio rápido en pacientes sin ninguna enfermedad hepática previa" (Montrief \& Koyfman, 2019). La progresión de hepatitis crónica a insuficiencia hepática crónica depende de la etiología subyacente.

Se estima que menos del $1 \%$ de los pacientes con hepatitis A crónica y aproximadamente el $1 \%$ de los pacientes con hepatitis B crónica progresarán a IHA. En contraste, del 20 al 40\% de los pacientes con hepatitis E crónica progresan a IHA en los países en desarrollo. Aproximadamente el 69\% de los pacientes con hepatitis autoinmunitaria crónica y grave progresan a ALF, y aproximadamente el $2 \%$ de los casos de ALF resulta de la enfermedad de Wilson (Kwong \& Meyerson, 2019).
La etiología específica de la insuficiencia hepática crónica también es un predictor esencial de la recuperación espontánea. "Aproximadamente el $75 \%$ de los pacientes se recupera espontáneamente de la falla inducida por acetaminofén (paracetamol), pero solo alrededor del $40 \%$ se recupera espontáneamente de otras causas" (Stravitz \& Lee, 2019)

Los pacientes con insuficiencia hepática crónica deben considerarse para un trasplante de hígado y deben ser trasladados de inmediato a los centros de trasplante. "Existen varias herramientas de cribado de pronóstico para la IHA y la evaluación para el trasplante de hígado de emergencia" (Montrief \& Koyfman, 2019).

\section{Conclusiones}

Dadas las exhaustivas condiciones que pueden causar hepatitis aguda, el manejo de esta condición clínica requiere un enfoque de equipo interprofesional y colaborativo que ayude a hacer un diagnóstico oportuno que resulte en un manejo adecuado. La presentación clínica de la hepatitis crónica en los pacientes puede variar desde estar asintomáticos con resultados de pruebas bioquímicas hepáticas anormales incidentales hasta estar críticamente enfermos con signos de insuficiencia hepática aguda. En la práctica clínica, la hepatitis crónica es detectada principalmente por médicos de atención primaria, médicos de medicina de emergencia e internistas. Según la etiología y la gravedad, es posible que otros especialistas deban participar en el equipo de atención, incluidos gastroenterólogos, hepatólogos, farmacéuticos, personal de enfermería, toxicólogos, especialistas en enfermedades infecciosas, cirujanos de trasplantes.

En algunos casos de hepatitis crónica es causada por sobredosis de drogas o abuso de drogas, es importante la participación de expertos en salud conductual y profesio- 
nales en abuso de sustancias. Los farmacéuticos clínicos deben revisar minuciosamente el perfil de medicación del paciente y ayudar con la dosificación adecuada de la medicación para evitar más agresiones por hepatotoxicidad por medicamentos e interacciones medicamentosas.

\section{Bibliografía}

Agrawal, S., Dhiman, R., \& Limdi, J. (2016). Evaluation of abnormal liver function tests. . Postgrad Med J , 92 (1086), 223-34.

Bolívar, J. (2015). Investigación Documental. México. Pax.

Caballeríaa, J., Caballeríaa, A., \& Parésa, M. (2000). Enfermedad hepática alcohólica. Rev Medicina Integral , 35 (10), 474-480.

Castro, J. (2016). Técnicas Documentales. México. Limusa.

Davila, A. (2015). Diccionario de Términos Científicos. . Caracas: Editorial Oasis.
Hosseini, N., Shor, J., \& Szabo, G. (2019). Alcoholic Hepatitis: A Review. . Alcohol Alcohol , 54 (4), 408416.

Kwong, S., \& Meyerson, C. (2019). Acute hepatitis and acute liver failure: Pathologic diagnosis and differential diagnosis. . Semin Diagn Pathol , 36 (6), 404-414.

Montrief, T., \& Koyfman, A. (2019). Acute liver failure: A review for emergency physicians. Am J Emerg Med , 37 (2), 329-337.

Semin Diagn Pathol. (2019). Enfermedad de Guindi M. Wilson. . Seminario Diagnostico Pathol, 36, págs. 415-422.

Sosa, A., \& Arrese, R. (25 de May de 2017). Insuficiencia Hepatica Aguda. Recuperado el 08 de Feb de 2021, de Universidad Catolica de Chile: https://medicina.uc.cl/publicacion/insuficiencia-hepatica-aguda/

Stravitz, R., \& Lee, W. (2019). Acute liver failure. Lancet. , 394 (10201), 869-881.

Woreta, T. (2014). valuation of abnormal liver tests. Med Clin North Am. , 98 (1), 1-16.

\section{CITAR ESTE ARTICULO:}

Pico Tagle, A. N., Chávez Heredia, L. G., Moreira Moreira, L. A., \& Palacios Saltos, J. E. (2020). Causas y consecuencias de los pacientes diagnosticados con hepatitis crónica. RECIMUNDO, 4(4), 539-547. https://doi.org/10.26820/ recimundo/4.(4).octubre.2020.539-547

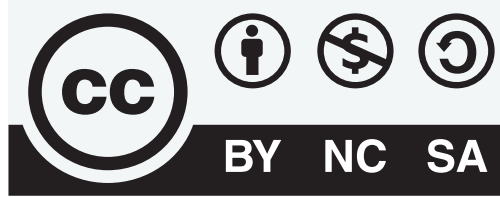

